\title{
Mais à quoi jouent donc BRCA1 et BRCA2 ?
}

Le monde scientifique a eu à faire face, depuis deux années à une avalanche d'informations souvent contradictoires sur le rôle des produits des anti-oncogènes BRCA1 et BRCA2. Tout d'abord, plusieurs articles ont proposé que les protéines BRCA1 et BRCA2 pouvaient appartenir à la famille des granines et agir au niveau extra-cellulaire, se comportant peut-être comme des facteurs diffusibles inhibiteurs de la prolifération cellulaire $\left(\mathrm{m} / \mathrm{s} n^{\circ} 6 / 7\right.$, vol. 12, p. 812). Cependant, ces informations devaient être démenties et plusieurs auteurs démontraient ensuite très clairement que ces protéines étaient à localisation intranucléaire $(\mathrm{m} / \mathrm{s}$ $n^{\circ} 11$, vol. 12, p. 1271), et semblaient plutôt agir en tant que facteur de transcription, possédant d'ailleurs des domaines d'activation transcriptionnelle $\left(m / s n^{\circ} 3\right.$, vol. 13, p. 418). Couplés à un domaine de liaison à l'ADN exogène, le domaine d'activation de BRCA1 permet de stimuler la transcription de gènes tests. En outre, plusieurs mutations du gène BRCA1 intéressent le domaine d'activation et altèrent son activité transcriptionnelle. Une équipe britannique de Cambridge vient également d'identifier dans la protéine BRCA2 un tel domaine d'activation présentant des similitudes de séquences avec un domaine d'activation c-Jun [1]. Cependant, c'est vers une piste totalement différente qu'oriente toute un série de travaux démontrant que les produits des gènes $B R C A$ peuvent former un complexe avec la protéine RAD51 humaine[2, 3]. Les gènes Rad51 sont, dans des cellules eucaryotes, les équivalents du gène RecA de E. coli et interviennent dans des cassures double brin, et dans la recombinaison associée à la réparation [4]. D'ailleurs, chez la souris, Brcal et Rad51 sont co-localisés au niveau des complexes synaptonémaux (situés à la jonction entre les chromosomes méiotiques et nécessaires à la recombinaison homologue). L'inactivation par recombinaison homologue des gènes Brcal et Brca 2 chez la souris aboutit, dans les deux cas, à une mortalité embryonnaire précoce $[5,6]$. De plus, les tissus des embryons Brca2 $2^{-1-}$ montrent une hypersensibilité aux radiations ionisantes, ce qui augmente encore les similitudes entre le phénotype de ces animaux et celui de souris déficientes en Rad51 [7].

Tout ces résultats suggèrent que si les protéines BRCA1 et BRCA2 sont bien des facteurs nucléaires, elles pourraient intervenir plus dans la réparation de l'ADN que vraiment dans la transcription [4]. Cela est assez cohérent avec le phénotype des femmes porteuses d'une mutation hétérozygote constitutive de l'un des deux gènes, BRCA1 ou BRCA2. En effet, si certaines mutations du gène $B R C A 1$ ont une pénétrance élevée (de l'ordre de $80 \%$ ), les cancers du sein ou de l'ovaire qui apparaissent peuvent être tardifs; par ailleurs, d'autres mutations, et notamment du gène $B R C A 2$, ont une pénétrance beaucoup plus faible. Pour Kinzler et Vogelstein (Baltimore, MD, USA) cela rapprocherait plus les gènes BRCA1 et BRCA2 de la famille des anti-oncogènes caretakers que de celles des gatekeepers [4]. Les caretakers correspondent à une famille de gènes intervenant dans la stabilité du matériel génétique, parmi lesquels ont peut classer les gènes mutés dans les formes familiales non polyposiques du cancer du côlon (codant pour des enzymes de la réparation des mésappariements d'ADN [8]), le gène $A T M$ de l'ataxie télangiectasie, les gènes du xeroderma pigmentosum. La pénétrance des mutations de ces gènes est faible parce qu'elle doit être suivie d'au moins trois autres événements génétiques pour aboutir à un cancer: mutation du second allèle du gène caretaker impliqué, complétant le déficit en système de réparation, puis mutations succesives des deux allèles d'anti-oncogènes appartenant à la famille des gatekeepers [4]. A l'inverse, la mutation d'un allèle de gène gatekeeper n'a besoin d'être associée qu'à l'inactivation de l'autre allèle pour permettre le développement d'un cancer. Ces gènes gatekeepers codent pour les anti-oncogènes du type $\mathrm{Rb}$ (de susceptibilité au rétinoblastome), APC (produit du gène de la polypose colique familiale), NF1 (impliqué dans la neurofibromatose de type 1), etc. Les notions selon lesquelles les produits des gènes BRCA1 et BRCA2 sont des facteurs de transcription et des protéines intervenant dans un complexe de réparation ne sont pas totalement contradictoires. En effet, des exemples existent où des protéines formant un complexe avec l'ADN peuvent intervenir dans la réplication de l'ADN, sa transcription et sa réparation: il suffit pour s'en convaincre de se reporter à la saga des sous-unités du facteur de transcription TFII$\mathrm{H}$, plusieurs fois présentée à nos lecteurs par Jean-Marc Egly (Strasbourg, France) [9, 10]. Reste à savoir laquelle de ces fonctions est indispensable à la protection contre un développement tumoral. 
Dans l'hypothèse où le rôle des gènes BRCA1 et BRCA2 dans la réparation serait démontrée, il resterait alors à déterminer la nature des altérations génétiques «d'aval »... c'est-à-dire l'identité des gènes gatekeepers affectés du fait de la mutation de BRCA1 ou de BRCA2.

\section{Axel Kahn}

Inserm U. 129, ICGM, 24, rue du Faubourg-Saint-Jacques, 75014 Paris, France.

1. Milner J, Ponder B, Hughes-Davies L, Seltmann M, Kouzarides T. Transcriptional activation functions in BRCA2. Nature 1997; 286: 772-3.

2. Scully R, Chen J, Plug A, Xiao Y, Weaver D, Feunteun J, Ashley T, Livingston DM. Association of BRCA1 with Rad51 in mitotic and meiotic cells. Cell 1997; 88: 265-75.

3. Sharan SK, Morimatsu M, Albrecht U, Lim DS, Regel E, Dinh C, Sands A, Eichele G, Hasty P, Bradley A. Embryonic lethality and radiation hypersensitivity mediated by Rad51 in mice lacking Brca2. Nature 1997; 386 : 804-10.

4. Kinzler KW, Vogelstein B. Cancer susceptibility genes: gatekeepers and caretakers. Nature 1997; 386: 761-3.

5. Hakem R, de la Pomba JL, Sirard C, Mo R, Woo M, Hakem A, Wakeman A, Potter J, et al. The tumor suppressor gene Brcal is required for embryonic cellular proliferation in the mouse. Cell. 1996; 85 : 1009-23.

6. Liu CY, Flesken-Nikitin A, Li S, Zeng Y, Lee WH. Inactivation of the mouse Brcal gene leads to failure in the morphogenesis of the egg cylinder in early postimplantation development. Genes Dev 1996; 10: 1835-43.

7. Lim DS, Hasty T. A mutation in mouse Rad51 results in an early embryonic lethal that is suppressed by a p53 mutation. Mol Cell Biol 1996; 16: 7133-43.

8. Radman M, Taddei F, Halliday J. Correction des erreurs dans l'ADN: de la génétique bactérienne aux mécanismes de prédisposition héréditaire aux cancers chez l'homme. Med Sci 1994; 10: $1024-30$.

9. Schaeffer L, Egly J. BTF2/TFIIH, un facteur de transcription et réparation impliqué dans des maladies de la réparation de l'ADN. Med Sci 1994; 10: $973-8$.

10. Roy R, Bergmann E, Egly J. TFIIH (BTF2), à l'interface de trois processus cellulaires: transcription, réparation et cycle cellulaire. $\mathrm{Med} \mathrm{Sci}$ $1995 ; 11: 879-82$.

\section{TIRÉS À PART}

A. Kahn.

\section{BRÈVES}

Activation lymphocytaire et signal Wnt: existe-t-il une connexion ? L'un des phénomènes les mieux connus de l'activation des lymphocytes $\mathrm{T}$ est la stimulation par le calcium de la protéine phosphatase calcineurine, aboutissant au transfert du facteur de transcription NF-AT dans le noyau et, ainsi, à la stimulation du gène codant pour l'interleukine-2. C'est cette étape qui est inhibée par l'immuno-suppresseur ciclosporine et les agents équivalents [1]. Les sérines déphosphorylées par la calcineurine activée sont la cible d'un complexe kinasique dont un important composant vient d'être caractérisé par l'équipe de Gérald R. Crabtree (Standford, CA, USA) [2]. Ces auteurs montrent en effet que la NF-AT kinase comporte la sérine/thréonine kinase GSK-3 (glycogène synthase 3). La surexpression de cette enzyme GSK-3 bloque l'activation des lymphocytes $\mathrm{T}$ et entraîne un maintien de NF-AT dans le cytoplasme. Comme nous l'avons vu récemment [3], la kinase GSK-3 est également inhibée en réponse au signal issu des facteurs de croissance Wnt, qui pourraient ainsi contribuer à des phénomènes d'activation cellulaire, facilitant la translocation nucléaire de NF-AT. Dans une mini-synthèse de ce même numéro de médecine/sciences (voir p. 872), Béatrice Romagnolo rappelle aussi qu'une isoforme de GSK-3, inhibée par la stimulation de la voie Wnt, stimule la dégradation de la $\beta$-caténine, de telle sorte que Wnt entraîne une accumulation de cette protéine qui peut être transférée dans le noyau sous la forme d'un complexe activateur de la transcription Tcf/LEF. Existe-t-il dans les lymphocytes $\mathrm{T}$ une synergie entre les gène activés par NFAT et Tcf/LEF?

[1. Baumann G, Borel J. Med Sci 1992; 8: 366-71.]

[2. Beals CR, et al. Science. 1997; 275: 1930-3.]

[3. Bellaïche Y, Perrimon N. Med Sci 1997; 13: 166-74.]
GÉNÉTIQUE

DE LA FERTLLTÉ

MASCULINE

COLLIOURE, FRANCE

4-6 septembre 1997

\section{Jeudi 4 septembre}

GENETIC CONTROL OF SPERMATOGENESIS

Genetic of sexual differenciation: C. Sultan (F)

Genetic control of spermatogenesis: N. Hecht (USA)

Mapping of the $Y$ : D. Page (USA)

Epidemiology of oligo and azoospermia : A. Spira (F)

Genetic aspects of flagellar dyskinesia, globozoospermia: C. Gagnon (C)

Urogenital dysgenesis and male infertility : P.N. Schlegel (USA)

Karyotype and oligospermia: A. Chandley (UK)

Heritability of sterility : $H$. Tournaye (B)

\section{Vendredi 5 septembre}

GENETIC OF THE SPERMATOZOA DNA packaging : $S$. Ward (USA) Detection and characterization of chromosome abnormalities: R. H. Martin (C) $X / Y$ separation : J.D. Schulman (USA) Oxydative damages of chromatin: D. Sakkas (S)

\section{SESSION DE POSTERS}

TABLE RONDE ÉTHIQUE McDonough (USA), M. Serres (F), F. Collins (USA), A. Kahn (F)

\section{Samedi 6 septembre}

ROLE OF SPERMATOZOA IN EMBRYOGENESIS

Paternal effects on early embryogenesis : L. Janny (F)

Paternal inheritance of the centrosome : G. Schatten (USA)

Gene imprinting : N. De Groot (IL)

Mitochondrial DNA : J. Cummins (Aus)

$$
\text { Renseignements : }
$$

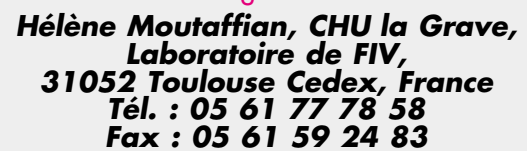

